\title{
ASSESSMENT OF INTRARENAL VASCULARIZATION IN DIFFUSE SYSTEMIC SCLEROSIS
}

\author{
Alexandru Caraba', Viorica Crisan ${ }^{2}$, Andreea Munteanu ${ }^{1}$, Ioan Romosan ${ }^{1}$ \\ ${ }^{I}$ Department of Internal Medicine, Victor Babes University of Medicine and Pharmacy, Timisoara \\ ${ }^{2}$ Emergency Clinical Hospital, Timisoara
}

\begin{abstract}
Systemic sclerosis is an autoimmune disease, characterized by fibrosis and vascular abnormalities, which determine the clinical manifestations and prognosis. The aim of this study was the assessment of intrarenal vascularization in diffuse systemic sclerosis patients and its correlations with nailfold capillaroscopy abnormalities.

Material and methods. The study was performed on 11 patients with diffuse systemic sclerosis without any renal abnormalities and 11 sex- and age-matched controls. In all patients were determined: glomerular filtration rate (GFR), proteinuria, urinary sediment, resistive (RI) and pulsatility (PI) index of interlobar renal arteries and the number of nailfold capillaries $/ \mathrm{mm}$. All the data were presented as mean \pm standard deviation. The statistically analysis was done using Pearson's test and Student's t-test, $p<0.05$ was considered statistically significant. Results. The mean age of diffuse systemic sclerosis patients was $46.90 \pm 4.98$ years, and the mean length of disease was $4.63 \pm 2.96$ years. GFR, RI, and PI were significantly lower in diffuse systemic sclerosis patients than in controls $(p<0.001)$. It was identified a strong correlation between nailfold capillaries density/mm and GFR $(r=0.719, p<0.05)$, RI $(r=-0.784, p<0.01)$, PI $(r=-0.748, p<0.01)$, and the mean length of the disease $(r=-0.85, p<0.001)$.

Conclusion. In patients with systemic sclerosis, intrarenal vascularization and glomerular filtration rate correlate with the capillaroscopic findings.

Keywords: diffuse systemic sclerosis, intrarenal vascularization, nailfold capillaroscopy
\end{abstract}

Systemic sclerosis (SSc) is an autoimmune multisystem disease, characterized by immune activation, autoantibody production, fibroblast dysfunction, extracellular matrix deposition, and vasculopathy (1). Its incidence is about 20 cases/ 1 million/year, rising in the last years. The SSc prevalence varies from 250 to 350 cases/million people $(2,3)$.

Major organ involvement (lung, kidney, heart, gastrointestinal tract) is associated with decreased survival in these patients (1). In their study, Steen and Medsger revealed that the 9-year survival in sclerodermic patients with major organs involvement is reduced at $39 \%$, compared with the patients without these comorbidities, in whom the survival is about $72 \%$ (4).

Renal involvement represents an important cause of morbidity and mortality in SSc patients. The spectrum of renal complications in SSc includes: sclero- derma renal crisis, normotensive scleroderma renal crisis, myeloperoxidase-antineutrophil cytoplasmic antibodies associated glomerulonephritis and vasculitis, penicillamine-associated renal disease, nephropathy induced by antiphospholipid antibodies, isolated urinary abnormalities (proteinuria, microscopic haematuria), isolated reduction in glomerular filtration rate. Another kidney involvement in scleroderma, less studied, is represented by abnormal increase of intrarenal resistance indices, without any clinical or biological signs (5). Evidence of renal disease may identify a subset of patients with scleroderma, who will experience significant morbidity and mortality (6). Subclinical renal impairment affects approximately $50 \%$ of scleroderma patients and may be associated with other vascular manifestations. Subclinical renal involvement rarely progresses to end-stage renal failure; however, Shanmu-

\footnotetext{
Correspondence address:

Alexandru Caraba, Department of Internal Medicine, Victor Babes University of Medicine and Pharmacy Timisoara

E-mail: alexcaraba@yahoo.com
} 
gam and Steen suggest that it may predict mortality in patients with other vasculopathic manifestations (5).

Microcirculation of patients with SSc is assessed at the nailfold skin of the fingers of the hands by means of nailfold capillaroscopy. Based on the capillaroscopic abnormalities, Cutolo classified the sclerodermic capillaroscopic pattern into three stages: early (few giant capillaries, few capillary microhaemorrhages, no evident loss of capillaries, and a relatively well-preserved capillary distribution), active (frequent giant capillaries, frequent capillary microhaemorrhages, moderate loss of capillaries, absence of or mildly ramified capillaries with slight disorganisation of the capillary architecture), and late (irregular enlargement of the capillaries, almost absent giant capillaries and microhaemorrhages, severe loss of capillaries with extensive avascular areas, ramified/bushy capillaries, and intense disorganisation of the normal capillary array) (7).

Intrarenal vascularisation may be assessed by means of Doppler ultrasonography of interlobar renal arteries, determining the resistive index (RI) and pulsatile index (PI) (8).

The aim of this study is represented by the assessment of intrarenal vascularization and its correlation with the nailfold capillaroscopy findings in diffuse SSc patients.

\section{MATERIAL AND METHODS}

The study was performed on a group of 11 patients with systemic sclerosis without clinical and biological renal abnormalities and 11 sex- and agematched controls. The diagnosis of SSc was established based on 2013 Classification Criteria for Systemic Sclerosis (9). Exclusion criteria were: isolated urinary abnormalities (microhaematuria and/or proteinuria), primary or secondary kidney diseases (glomerulonephritis, tubular-interstitial nephropathies, nephrolithiasis, renal cysts, vascular nephropathies), arterial hypertension, diabetes mellitus, heart failure, generalized atherosclerosis.

Renal function was assessed by means of glomerular filtration rate (GFR), proteinuria/24 hour, and urinary sediment. GFR was calculated using the formula:

GFR $=$ urinary creatinine $(\mathrm{mg} / \mathrm{dl}) \times$ urinary volume (ml) / serum creatinine (mg/dl) x 1440.

Serum and urinary creatinine were determined using Jaffe method, proteinuria by biuret method, and urinary sediment by Addis-Hamburger method.
Intrarenal vascularization was characterized by resistive (RI) and pulsatility (PI) indices of interlobar renal arteries. They were determined by means of Doppler ultrasonography, with Siemens Acuson X300 Ultrasound System, with convex 3,5 MHz probe.

Capillaroscopy is a non-invasive imaging technique that is used for in vivo assessment of the microcirculation. In our study, nailfold capillaroscopy was performed with USB Digital Microscope 2.0 mega Pixel Digital Camera.

Before starting this procedure, the patients took place in a room with a stable temperature of $20-22^{\circ} \mathrm{C}$ for at least 15 minutes, in order to avoid capillaries vasoconstriction, which can induce false positivity for avascular areas. The $2^{\text {nd }}, 3^{\text {rd }}, 4^{\text {th }}$ and $5^{\text {th }}$ fingers of both hands were examined. Giant capillaries, capillaries haemorrhages, avascular areas, and capillary architecture were the recorded nailfold capillaroscopic parameters $(10,11)$. Nailfold capillaries density $/ \mathrm{mm}$ was the parameter used in the statistical analysis.

All the data were presented as mean \pm standard deviation. The statistically analysis was done using Pearson's test and Student's t-test, $\mathrm{p}<0.05$ was considered statistically significant.

\section{RESULTS}

The group of patients with SSc was formed by 8 females and 3 males, with the mean age of $46.90 \pm$ 4.98 years. All the patients had diffuse form of SSc, with the mean length of evolution of $4.63 \pm 2.96$ years. Antinuclear antibodies were present in all the patients. The assessed parameters are presented in the Table 1 .

TABLE 1. Clinical and imagistic parameters assessed in sclerodermic patients

\begin{tabular}{|l|c|c|c|}
\hline Parameter & $\begin{array}{c}\text { Systemic } \\
\text { sclerosis } \\
\text { group }\end{array}$ & $\begin{array}{c}\text { Healthy } \\
\text { control } \\
\text { group }\end{array}$ & $p$ \\
\hline Age (years) & $46.90 \pm 4.98$ & $44.9 \pm 7.17$ & 0.456 \\
\hline Capillaries/mm & $6.25 \pm 2.35$ & $12.3 \pm 1.22$ & 0.0000026 \\
\hline $\begin{array}{l}\text { Proteinuria } \\
\text { (mg/24 hours) }\end{array}$ & $89.63 \pm 14.04$ & $94.27 \pm 11.26$ & 0.441 \\
\hline $\begin{array}{l}\text { Urinary sediment } \\
\text { (red blood } \\
\text { cells/min) }\end{array}$ & $527 \pm 251$ & $501 \pm 169$ & 0.401 \\
\hline GFR (ml/min) & $82.54 \pm 6.43$ & $101 \pm 5.76$ & 0.0000072 \\
\hline RI & $0.71 \pm 0.03$ & $0.62 \pm 0.02$ & 0.0000079 \\
\hline PI & $1.30 \pm 0.08$ & $1.13 \pm 0.02$ & 0.000053 \\
\hline
\end{tabular}


In the SSc group, the capillaries density/mm was significantly reduced than in controls (Fig. 1,2). Our study reveals a strong inverse correlation between the capillaries density and the mean length of disease evolution $(\mathrm{r}=-0.85, \mathrm{p}<0.001)$ (Fig. 3).

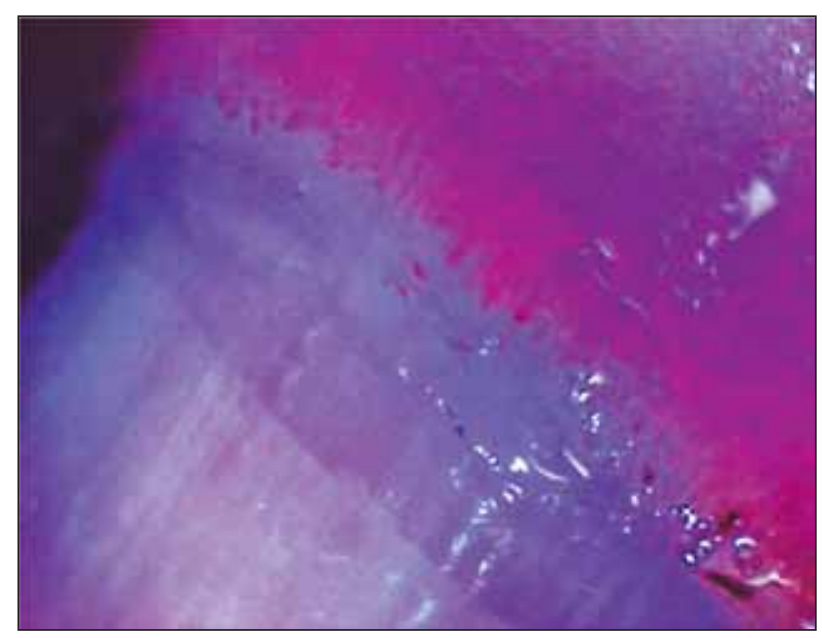

FIGURE 1. Nailfold capillaroscopy in systemic sclerosis patients (active pattern)

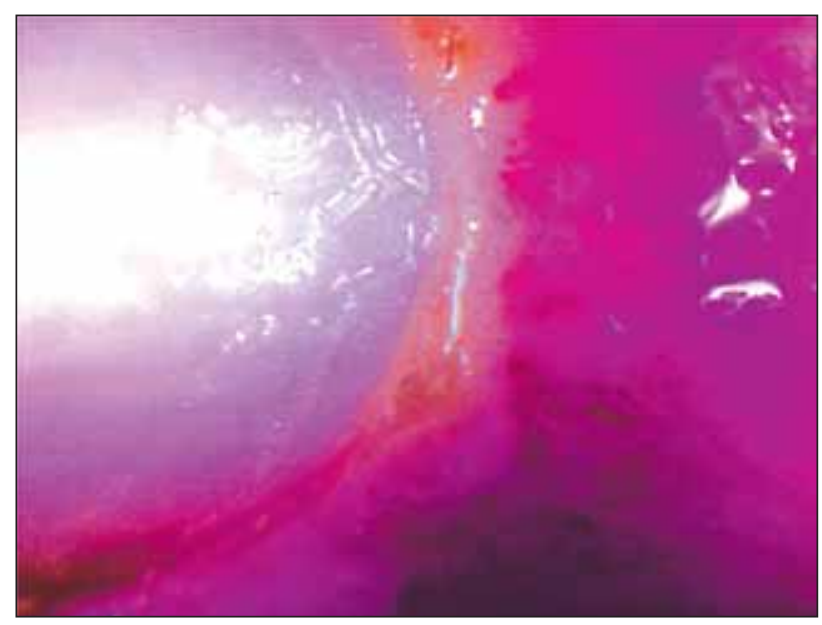

FIGURE 2. Nailfold capillaroscopy in systemic sclerosis patients (late pattern)
Proteinuria and urinary sediment didn't show any changes in sclerodermic group. But glomerular filtration rate was significantly reduced in these patients $(p=0.0000072)$. Resistive and pulsatility indices were higher in studied patients than in controls ( $p=0.0000079$ for RI, and $p=0.000053$ for PI), demonstrating the presence of intrarenal vasoconstriction (Fig. 4). These parameters were correlated with the capillaries density. We found a significant correlation between the capillaries density and GFR $(r=0.719, p=0.012)$. Other correlations were established between the capillaries density and RI $(\mathrm{r}=-0.784, \mathrm{p}=0.004)$, respective PI $(\mathrm{r}=-0.748$, $\mathrm{p}=0.008$ ). (Fig. 5, 6).

\section{DISCUSSION}

Vascular pathology represents one of the main factors involved in pathogenesis and organ dysfunction in SSc. Vascular involvement in SSc affects predominantly the microcirculation (arterioles and capillaries). Vascular dysfunction and disorganised microvasculature occur early in the disease evolution, and may predict the prognosis (3).

Sclerodermic vessels pathology shows concentric intimal proliferation, marked luminal obstruction, and lymphocyte infiltration (12). Microvasculature changes, such as those assessed by nailfold capillaroscopy, are seen in all involved organs (lungs, heart, kidneys, and gastrointestinal tract), demonstrating the widespread nature of capillary changes in this disease (13).

Renal involvement in SSc could exhibit several forms of different severity, from asymptomatic renal involvement to sclerodermic renal crisis. Trostle et al. reported that the asymptomatic renal pathology was identified from autopsy studies in $60-80 \%$ of

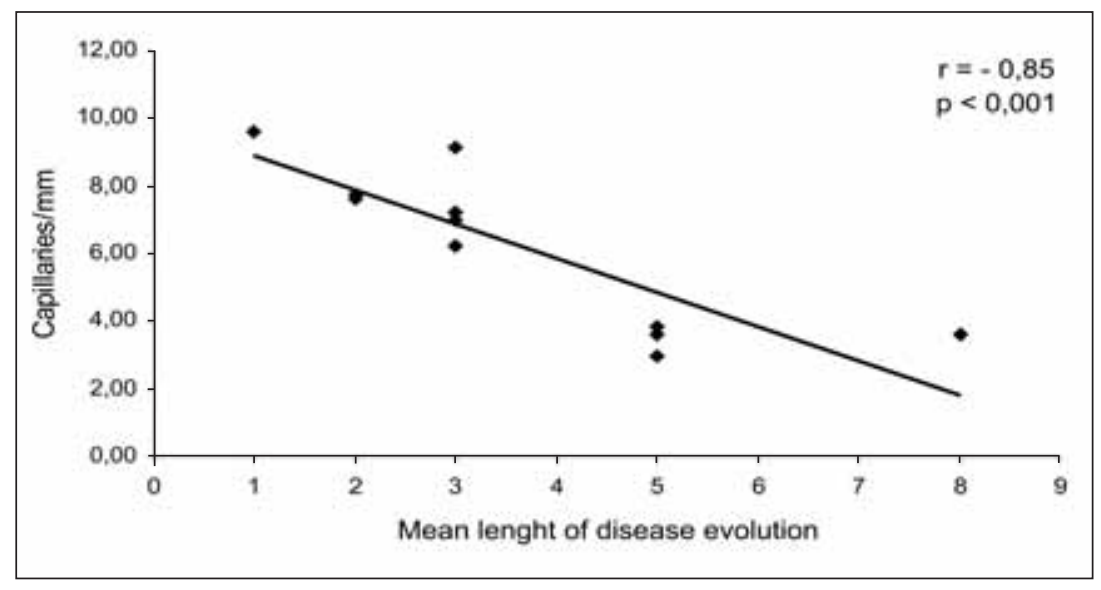

FIGURE 3. Correlation between the capillaries density and the mean length of disease evolution 


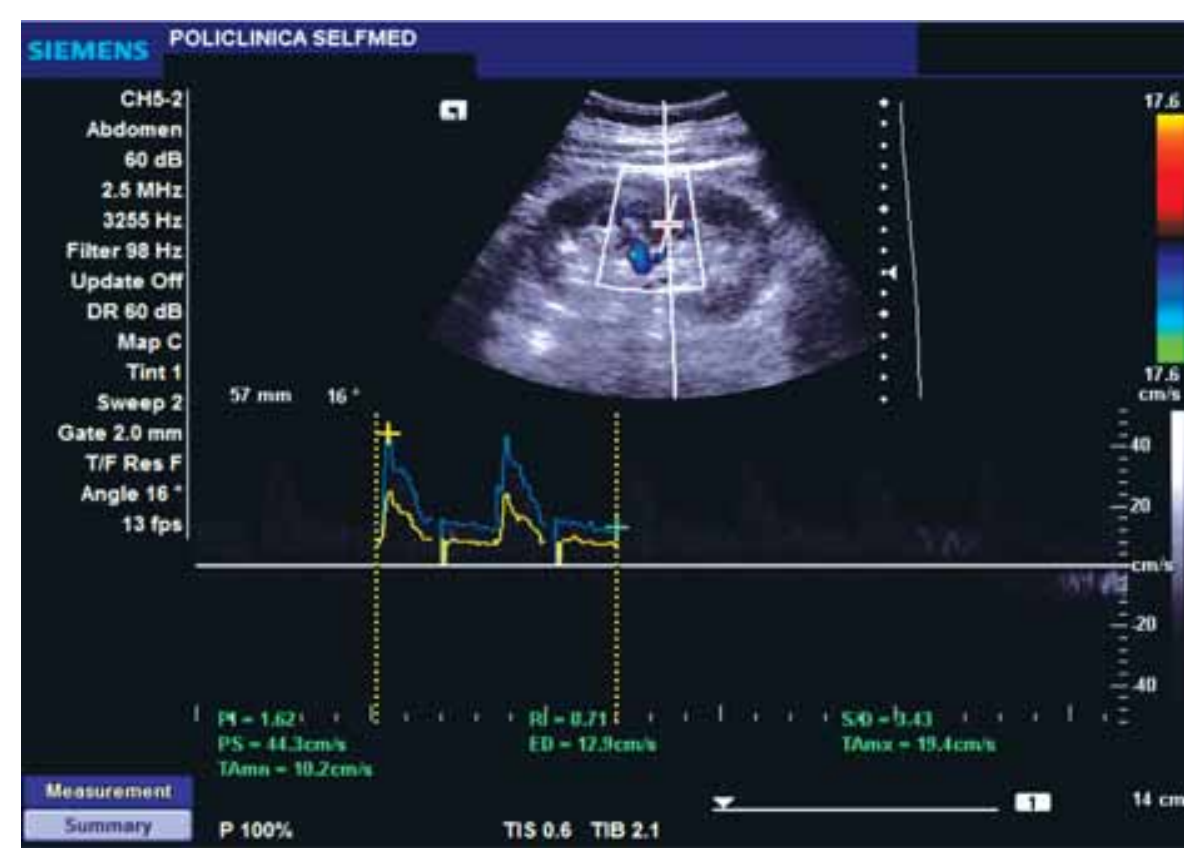

FIGURE 4. Intrarenal

vascularization in sclerodermic patients $(P I=1.62, R I=0.71)$
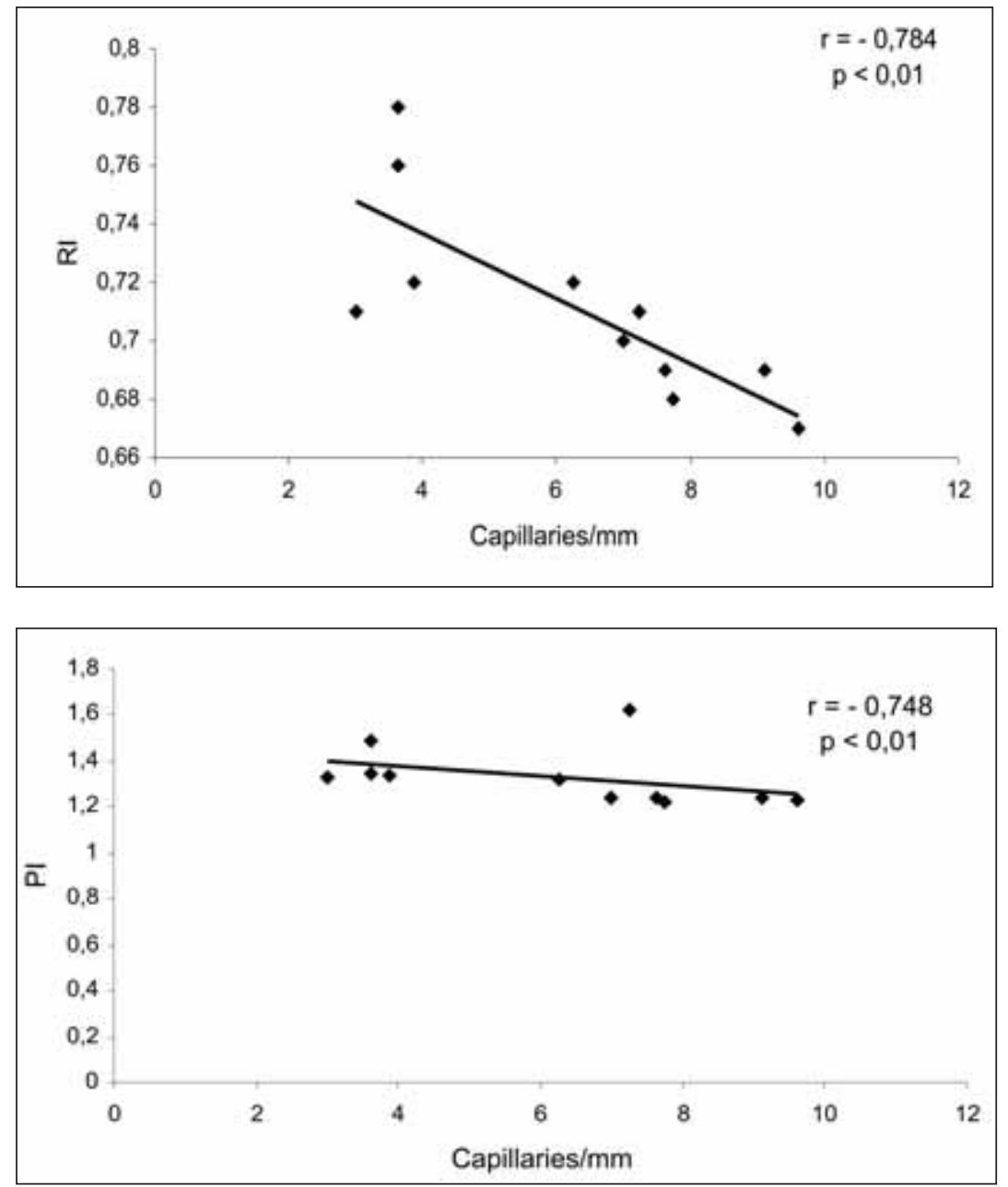

FIGURE 5. Correlation between the capillaries density and $R I$
FIGURE 6. Correlation between the capillaries density and PI sclerodermic patients. They demonstrated that the intimal thickening was present in small and medium-sized arteries of patients with diffuse SSc, but only in small arteries of limited SSc patients, com- pared with those in controls. The authors concluded that renal vascular structural changes represent an important feature in SSc, with major impact on prognosis (14). Histopathologic renal injury is common 
in scleroderma, even without clinically evident sclerodermic renal crisis (5).

In our study, GFR was reduced in scleroderma patients, compared to healthy controls $(\mathrm{p}<0.001)$. However, the lowest value of GFR wasn't below of $69 \mathrm{ml} / \mathrm{min}$. These values of GFR weren't accompanied by pathological proteinuria, urinary sediment, or elevated serum creatinine. Kingdon et al. showed that the impairment of renal function can be present in patients with diffuse SSc without clinical and biological sign of renal involvement (15). In another study, Scheja et al. identified that the decreased glomerular filtration rate was found in $11 \%$ of limited SSc and $8.6 \%$ of diffuse SSc patients (16). In their study, performed on 675 patients with diffuse SSc, Steen el al. reported reduced renal function in $32 \%$ of patients. During a period of 10 years, none of the patients developed severe renal failure, suggesting that the asymptomatic reduced of renal function is common in scleroderma, having a benign clinical course (17). It was revealed a correlation between GFR and capillaries density in SSc patients $(r=$ $0.719, p=0.012)$. This relation between GFR and capillaroscopic damage was described by Rosato et al. in their study (18).

Resistive and pulsatility indices of interlobar renal arteries represent markers of severity renal damage. They evaluate the intrarenal elasticity and compliance. Their elevated value identifies vascular and interstitial nephropathies, because glomeruli are only responsible for $10 \%$ of the intraparenchymal flow resistances. Intrarenal vessels remodeling leads to elevation of RI as renal vascular disease progresses $(8,18)$. In scleroderma patients, renal involvement appearance, even subclinical, is accompanied by the increase of these parameters (18). Our study reveals that these indices were increased in patients with SSc sclerosis, compared to controls ( $p<0,001)$. On the other hand, between these parameters and capillaries density, significant negative correlations were demonstrated $(\mathrm{r}=-0.784, \mathrm{p}=0.004$ for the correlation between capillaries density and RI, respectively $r=-0.748, p=0.008$ for the correlation between capillaries density and PI). Rosato et al. showed that the intrarenal indices were significantly increased in sclerodermic patients than in controls. The authors revealed that these indices increased with the progression of capillaroscopic damage progression (18). Rivolta et al. showed that in sclerodermic patients, RI was significantly increased than in controls (19). Aikimbaev et al. demonstrated the increase of Doppler indices of intrarenal vascular resistance in sclerodermic patients, compared with healthy controls, the values of these indices correlating with the disease duration, the age of patients and the plasma renin activity (20). Increased intrarenal resistive index signifies the presence of renal vascular involvement and correlates with GFR and digital microvascular damage in SSc patients (5).

In SSc, some of types of renal involvement do not have an immediate clinical significance. However, in the presence of severe capillaroscopic damage, intrarenal vascularization assessment can show significant vasoconstriction, even in absence of clinical or biological signs of renal involvement (21).

\section{CONCLUSION}

In patients with diffuse SSc, assessment of intrarenal vascularization shows a vasoconstrictive pattern. Significant correlations were demonstrated between capillaroscopic findings and the indices which characterizes the intrarenal vascularization and glomerular filtration rate.

In this context, finding severe capillaroscopic damage in a patient with SSc should be followed by the assessment of renal vascular involvement, even in the absence of any clinical or biological signs.

\section{$\overline{\text { REFERENCES }}$}

1. Shanmugam V.K., Steen V.D. Renal manifestations in scleroderma: evidence for subclinical renal disease as a marker of vasculopathy. Int J Rheumatol 2010, article ID 538589, 2010. doi:10.1155/2010/538589

2. Hachulla E., Czirjak L. (ed.). Textbook on Systemic Sclerosis. London: BMJ Publishing Group, 2013

3. Pattanaik D., Brown M., Postlethwaite A.E. Vascular involvement in systemic sclerosis (scleroderma). J Inflamm Res 2011; 4: 105-125
4. Steen V.D., Medsger T.A. jr. Severe organ involvement in systemic sclerosis with diffuse scleroderma. Arthritis Rheum 2000; 43(11): 2437-2444

5. Shanmugam V.K., Steen V.D. Renal disease in scleroderma: an update on evaluation, risk stratification, pathogenesis and management. Curr Opin Rheumatol 2012; 24(6): 669-76

6. Bulpitt K.J., Clements P.J., Lachenbruch P.A., et al. Early undifferentiated connective tissue disease: III. Outcome and 
prognostic indicators in early scleroderma (systemic sclerosis). Ann Intern Med 1993; 118(8): 602-9

7. Cutolo M., Sulli A., Secchi M.E., Paolino S., Pizzorni C. Nailfold capillaroscopy is useful for the diagnosis and follow-up of autoimmune rheumatic diseases. A future tool for the analysis of microvascular heart involvement? Rheumatology 2006; 45 (suppl 4): iv43-iv46

8. Dudea S.M., Badea R.I. Ultrasonografie vasculară. Bucureşti: Editura Medicală, 2004

9. van den Hoogen F., Khanna D.,Fransen J., et al. 2013 Classification Criteria for Systemic Sclerosis: an American College of Rheumatology/European League against Rheumatism collaborative initiative. Ann Rheum Dis 2013; 72(11): 1747-55

10. Cutolo M., Pizzorni C., Secchi M.E., Sulli A. Capillaroscopy. Best Pract Res Clin Rheumatol 2008; 22: 1093-108

11. Cutolo M. Atlas of capillaroscopy in rheumatic diseases. Milano: Elsevier, 2011

12. Trojanowska M. Cellular and molecular aspects of vascular dysfunction in systemic sclerosis. Nat Rev Rheumatol 2010; 6(8): 453-460

13. Grassi W., Core P., Carlino G., Blasetti P., Cervini M. Labial capillary microscopy in systemic sclerosis. Ann Rheum Dis 1993; 52(8): 564-569

14. Trostle D.C., Bedetti C.D., Steen V., et al. Renal vascular histology and morphometry in systemic sclerosis. A case-control autopsy study. Arthritis Rheum 1988; 31(3): 393-400
15. Kingdon E.J., Knight C.J., Dustan K. et al. Calculated glomerular filtration rate is a useful screening tool to identify scleroderma patients with renal impairment. Rheumatology 2003; 42 (1): 26-33

16. Scheja A., Bartosik I., Wuttge D.M., Hesselstrand R. Renal function is mostly preserved in patients with systemic sclerosis. Scand J Rheumatol 2009; 38(4): 295-298

17. Steen V.D., Syzd A., Johnson J.P., Greenberg A., Medsger T.A. jr. Kidney disease other than renal crisis in patients with diffuse scleroderma. J Rheumatol 2005; 32 (4): 649-655

18. Rosato E., Gigante E., Barbano B., et al. Intrarenal hemodynamic parameters correlate with glomerular filtration rate and digital microvascular damage in patients with systemic sclerosis. Semin Arthritis Rheum 2012; 41(6): 815-21

19. Rivolta R., Mascagni R., Berruti V., et al. Renal vascular damage in systemic sclerosis patients without clinical evidence of nephropathy. Arthritis Rheum 1996; 39(6): 1030-4

20. Aikimbaev K.S., Canataroglu A., Ozbek S., Usal A. Renal vascular resistance in progressive systemic sclerosis: evaluation with duplex Doppler ultrasound. Angiology 2001; 52(10): 697-701

21. Denton C.P. Renal manifestations of systemic sclerosis-clinical features and outcome assessment. Rheumatology 2008; 47 (suppl 5): 54-56 